\title{
Deletion of Dicer blocks osteogenic differentiation via the inhibition of Wnt signalling
}

\author{
HONG-YAN WU ${ }^{1}$, RUI BI ${ }^{1}$, TING $\mathrm{SUN}^{2}$ and FEI XIE ${ }^{1}$ \\ ${ }^{1}$ Pharmacy Department, Tangshan People's Hospital, Tangshan, Hebei 063001; ${ }^{2}$ Department of Clinical Pharmacology, \\ Fourth Hospital of Hebei Medical University, Shijiazhuang, Hebei 050011, P.R. China
}

Received March 20, 2018; Accepted January 29, 2019

DOI: $10.3892 / \mathrm{mmr} .2019 .9941$

\begin{abstract}
Micro (mi)RNAs are small, non-coding RNAs and have been reported to have important roles in the epigenetic control of bone development. miRNAs markedly regulate osteoblast differentiation through stages of maturation as well as the activities of osteogenic signaling pathways. Dicer is an important endoribonuclease that regulates miRNA maturation. Previous studies have demonstrated that Dicer deletion decreases fetal survival and bone formation, while excision in differentiated osteoblasts increases bone mass. However, the underlying molecular mechanisms remain unclear. In the present study, whether the deletion of Dicer affects Wnt signaling, which exhibits important roles during osteogenesis, was investigated. Bone marrow stromal cells (BMSCs) were used as an osteogenic model. Dynamic changes of seven Wnt genes and downstream T-cell factor 1 (Tcf-1)/lymphoid enhancing binding factor were observed during the osteogenic differentiation of BMSCs, which revealed different roles at early and late differentiation stages. Following the stable knockdown of Dicer in BMSCs using lentiviral short hairpin RNA, osteogenic differentiation was blocked, and the levels of important osteogenic differentiation markers (runt related transcription factor 2 and alkaline phosphatase) were markedly inhibited. Furthermore, stage specific regulation of Wnt genes in Dicer-deficient BMSCs was investigated in the present study. At the early differentiation stage (days 5-7), knockdown of Dicer led to the inhibition of Wnt1, Wnt7 and Wnt10b, as well as the upregulation of Wnt4, Wnt10a and Tcf-1. At the late stage of differentiation (days 14-21), knockdown of Dicer significantly suppressed the expression levels of all of the included Wnt genes as well as Tcf-1, with the exception of Wnt10a. The upregulation of Wnt10a following the deletion of Dicer was maintained throughout all stages of differentiation.
\end{abstract}

Correspondence to: Dr Hong-Yan Wu, Pharmacy Department, Tangshan People's Hospital, 65 Victory Road, Tangshan, Hebei 063001, P.R. China

E-mail: why130@hotmail.com

Key words: bone marrow stromal cells, osteogenic differentiation, Dicer, Wnt signalling, osteoblast, Wnt10a, microRNA
In addition, differential regulation of Wnt genes and Tcf-1 were revealed to be associated with dynamic changes in their expression levels during osteogenic differentiation. Furthermore, the four putative Wnt10a-targeting miRNAs were investigated in the present study, and the results demonstrated that they were upregulated during osteogenic differentiation, which suggested that inhibition of Wnt10a may be an important factor associated with osteogenic differentiation. In conclusion, the present study investigated the mechanism underlying the regulation of Wnt signalling by Dicer during osteogenesis, and identified potential miRNAs targeting the components of Wnt signalling influenced by Dicer. Collectively, the present study identified the association between Dicer and Wnt signalling during bone development.

\section{Introduction}

Osteogenic signaling pathways and transcription factors tightly regulate normal skeletal development and renewal of bone $(1,2)$. The important role of Wnt signaling during bone formation has been well established. Numerous human genetic studies have revealed that mutations in Wnt proteins or co-receptors result in osteoporosis, osteosclerosis and increased fracture risk (3-6). In agreement with such results in humans, genetic studies in mice have demonstrated similar roles of Wnt signaling in bone formation (7-9). Wnt proteins are a family of secreted glycoproteins, and associated signals are transduced by the binding of a Wnt protein to the frizzled receptor and co-receptor [such as low-density lipoprotein receptor related protein (LRP)5 and LRP6], which activates numerous distinct intracellular signaling cascades (10-13). The most well characterized canonical Wnt pathway is the $\beta$-catenin dependent pathway. The Wnt/ $\beta$-catenin signaling pathway is an evolutionarily conserved developmental signaling cascade that has an important role in regulating organ development and tissue homeostasis (14). $\beta$-catenin can interact with the lymphoid enhancing binding factor (Lef)/T cell factor 1 (Tcf-1) family of high mobility group-type transcription factors to induce the expression of target genes (15-18).

Gene regulatory mechanisms are essential for bone formation, including the epigenetic regulation of genes and post-translational biochemical modifications of protein activity. An increasing number of studies have revealed that micro (mi)RNAs, which are small, non-coding RNAs, 
have essential roles in the epigenetic control of bone development (19). miRNAs dynamically regulate osteoblast differentiation through stages of maturation and mediate the activities of osteogenic signaling pathways (20). Dicer, an endoribonuclease, is responsible for the processing of the mature miRNA duplex (21). Dicer deletion in mesenchymal progenitors decreases fetal survival and bone formation in mice, whereas excision in differentiated osteoblasts increases bone mass in adult mice (22). The conditional deletion of Dicer in mesenchymes by Wnt1-Cre, or in the epithelium by shh-Cre, revealed markedly different defects in tooth development (23), which suggests that deficiencies in mature miRNAs have the same effect on normal bone homeostasis when compared with miRNAs.

Although numerous miRNAs have been identified to target Wnt signaling components in osteoblasts (24-28), it has not been established whether miRNAs represent antagonists or activators of Wnt signaling during bone formation. In the present study, Dicer was knocked down in mouse bone marrow stromal cells (BMSCs) in order to establish an osteoprogenitor cell model with miRNA production deficiency. The results revealed that significant inhibition of osteogenic differentiation was associated with the differential regulation of numerous Wnt signalling components via the inhibition of Dicer in BMSCs. In contrast, the mechanism regulating the differentiation of osteoblast cells (MC3T3-E1) was identified to be different from BMSCs. The results of the present study also suggested that Wnt4 and Wnt10a may have more important roles during osteogenic differentiation compared with other Wnt genes. Furthermore, five putative Wnt10a-targeting miRNAs were demonstrated to be significantly upregulated during osteoblast differentiation.

\section{Materials and methods}

Cell culture. MC3T3-E1 are mouse osteoblasts (American Type Culture Collection, Manassas, VA, USA) and were cultured in $\alpha$-minimal essential medium (MEM) supplemented with $10 \%$ fetal bovine serum (FBS; Atlanta Biologicals, Inc., Atlanta, GA, USA). NIH3T3 cells are mouse fibroblasts (American Type Culture Collection) and were maintained in Dulbecco's modified Eagle's medium (Invitrogen; Thermo Fisher Scientific, Inc., Waltham, MA, USA) supplemented with $10 \%$ FBS (Atlanta Biologicals, Inc.). Cells were cultured in a humidified incubator at $37^{\circ} \mathrm{C}$ with $5 \% \mathrm{CO}_{2}$ (Thermo Fisher Scientific, Inc.). In order to investigate cell differentiation, MC3T3-E1 cells were incubated at $100 \%$ confluency in the aforementioned medium containing $10 \mathrm{mM} \beta$-glycerol phosphate and $50 \mu \mathrm{g} / \mathrm{ml}$ ascorbic acid (Sigma-Aldrich; Merck KGaA, Darmstadt, Germany) and fresh medium was added every other day, as previously reported (29).

Isolation of BMSCs. BMSCs were isolated from the femurs and tibias of five female and five male C57BL/6 mice (age, 6-8 weeks; weight, 20 \pm 2 g). All mice were purchased from and housed in a designated and government-approved animal facility of The Fourth Hospital of Hebei Medical University (Shijiazhuang, China). The mice were maintained at $21 \pm 2^{\circ} \mathrm{C}$, with $40-60 \%$ relative humidity, in a 12-h light/dark cycle and with free access to food and water. The present study was granted ethical approval by The Fourth Hospital of Hebei Medical University. BMSCs were prepared as previously reported (30). Soft tissues were disassociated from tibias and femurs. Bones were scrubbed and washed to remove impurities with complete $\alpha$-MEM medium on ice. A 23-gauge needle was inserted into the bone cavity to extract the bone marrow. Syringes with 20-gauge needles were used to dissociate cell clumps in the crude suspension. Following this, erythrocytes were eliminated using hypotonic lysis buffer and the collected cells were then resuspended in MSC growth media [ascorbic acid-free $\alpha$-MEM medium supplemented with $1 \mathrm{X}$ penicillin/streptomycin and $18 \%$ FBS (Hyclone; GE Healthcare)]. Single cell suspension was plated at a density of $1.5 \times 10^{5}$ cells per $10 \mathrm{~cm}$ culture plate for the expansion of the MSCs. BMSCs ranging from passages 6-10 were used for all subsequent experiments.

Transduction of Dicer short hairpin (sh)RNA and osteogenic differentiation in BMSCs. In total, $6 \mu \mathrm{g}$ lentiviral plasmid encoding for shRNAs against mouse Dicer1 (cat. no. MSH039774 GeneCopoeia, Inc., Rockville, MD, USA) or containing a scramble shRNA (cat. no. CSHCTR001-LVRH1GP; GeneCopoeia, Inc.) were co-transfected with $4 \mu \mathrm{g}$ psPAX2 (Addgene, Inc., Cambridge, MA, USA) and $2 \mu \mathrm{g}$ pMD2.G (Addgene, Inc.) packaging plasmids into 293FT cells (American Type Culture Collection) using Lipofectamine ${ }^{\circledR} 3000$ transfection reagent (Invitrogen; Thermo Fisher Scientific, Inc.) (30). Following a total of $48 \mathrm{~h}$ post-transfection, the viral particle-containing culture medium was collected. The medium was subsequently concentrated and $0.5 \mathrm{ml}$ lentiviral suspension $\left(1 \times 10^{8} \mathrm{IU} / \mathrm{ml}\right.$; multiplicity of infection, 100) was used to infect BMSCs in the presence of $5 \mu \mathrm{g} / \mathrm{ml}$ polybrene (Sigma-Aldrich; Merck KGaA), and the infected cells were then selected using puromycin (Sigma-Aldrich; Merck KGaA). The infection efficiency was investigated by determining Dicer mRNA expression levels by reverse transcription-quantitative polymerase chain reaction (RT-qPCR). Intact BMSCs and infected BMSCs were then cultured at $37^{\circ} \mathrm{C}$ in $\alpha$-MEM with $50 \mu \mathrm{g} / \mathrm{ml}$ ascorbic acid and $10 \mathrm{mM} \beta$-glycerol phosphate (Sigma-Aldrich; Merck KGaA) in order to induce osteoblast differentiation for 21 days. In order to perform miRNA analysis and RT-qPCR, cells were harvested at the indicated days (day 5, 7, 14 and or 21).

RNA isolation and RT-qPCR. RNA was exracted from cells (BMSCs, MC3T3 and NIH3T3) using TRIzol ${ }^{\circledR}$ reagent (Invitrogen; Thermo Fisher Scientific, Inc.). Following purification using Direct-zol RNA miniprep kit (Zymo Research Corp., Irvine, CA, USA), $1 \mu \mathrm{g}$ RNA was reverse transcribed using the qScript cDNA SuperMix for total cDNA (thermocycling conditions: $25^{\circ} \mathrm{C}$ for $5 \mathrm{~min}, 42^{\circ} \mathrm{C}$ for $30 \mathrm{~min}$ and $85^{\circ} \mathrm{C}$ for $5 \mathrm{~min}$ ) or qScript microRNA cDNA Synthesis Kit specifically for miRNA cDNA (thermocycling conditions: $60 \mathrm{~min}$ at $37^{\circ} \mathrm{C}, 5 \mathrm{~min}$ at $70^{\circ} \mathrm{C}, 20 \mathrm{~min}$ at $42^{\circ} \mathrm{C}$ and $5 \mathrm{~min}$ at $85^{\circ} \mathrm{C}$ ) purchased from Quantabio (Beverly, MA, USA) according to the manufacturer's protocol. TaqMan probes (Thermo Fisher Scientific, Inc.) and SYBRGreen (Thermo Fisher Scientific, Inc.) primers were used to determine relative gene expression (Tables I and II). The corresponding TaqMan and SYBR green mastermix (Thermo Fisher Scientific, Inc.) were used for the 
Table I. Primer sequences used to investigate gene expression and miRNA expression levels in the present study.

\begin{tabular}{|c|c|}
\hline Name & Primer sequences $\left(5^{\prime}-3^{\prime}\right)$ \\
\hline \multirow[t]{2}{*}{ Runx2 } & For: CGCCCCTCCCTGAACTCT \\
\hline & Rev: TGCCTGCCTGGGATCTGTA \\
\hline \multirow[t]{2}{*}{ ALP } & For: TTGTGCGAGAGAAAGGAGA \\
\hline & Rev: GTTTCAGGGCATTTTTCAAGGT \\
\hline \multirow[t]{2}{*}{$\mathrm{OC}$} & For: CTGACAAAGCCTTCATGTCCAA \\
\hline & Rev: GCGCCGGAGTCTGTTCACTA \\
\hline \multirow[t]{2}{*}{ Colla1 } & For: 5' CCCAAGGAAAAGAAGCACGTC \\
\hline & Rev: AGGTCAGCTGGATAGCGACATC \\
\hline \multirow[t]{2}{*}{ Dicer1 } & For: GGTGGTTCGTTTTGATTTGCC \\
\hline & Rev: GGCAGTGTTGATTGTGACTC \\
\hline \multirow[t]{4}{*}{ CEBPa } & For: CAAAGCCAAGAAGTCGGTGG \\
\hline & ACAA \\
\hline & Rev: TCATTGTGACTGGTCAACTC \\
\hline & CAGC \\
\hline \multirow[t]{2}{*}{$\operatorname{PPAR} \gamma$} & For: GAGTGTGACGACAAGATTTG \\
\hline & Rev: GGTGGGCCAGAATGGCATCT \\
\hline \multirow[t]{2}{*}{ FABP4 } & For: ATGTGCGACCAGTTTGTG \\
\hline & Rev: TTTGCCATCCCACTTCTG \\
\hline \multirow[t]{2}{*}{ GAPDH } & For: TCACCACCATGGAGAAGGC \\
\hline & Rev: GCTAAGCAGTTGGTGGTGCA \\
\hline
\end{tabular}

B, microRNAs

Name Primer sequences (5'-3')
mmu-miR-28

mmu-miR-149

mmu-miR-212

mmu-let-7b

mmu-let-7c

\section{AAGGAGCTCACAGTCTATTGAG TCTGGCTCCGTGTCTTCACTCCC ACCTTGGCTCTAGACTGCTTACT TGAGGTAGTAGGTTGTGTGGTT TGAGGTAGTAGGTTGTATGGTT}

For, forward; Rev, reverse; ALP, Akaline phosphatase; Colla1, collagen1a1; OC, osteocalcin; Runx2, runt related transcription factor 2; CEBP $\alpha, \operatorname{PPAR} \gamma$, peroxisome proliferator activated receptor- $\gamma$; FABP4, fatty acid binding protein 4 .

RT-qPCR reaction. All the related reagents and materials were purchased from Thermo Fisher Scientific, Inc. miRNA expression levels were detected using SYBR Green miRNA-specific primers (Table I). RNA isolation and RT-qPCR analyses were performed according to previously published protocol (31). RT-qPCR was performed using a 7300 sequence detection system (Invitrogen; Thermo Fisher Scientific, Inc.). The qPCR reaction was performed as follows: An initial incubation at $50^{\circ} \mathrm{C}$ for $2 \mathrm{~min}$ and at $95^{\circ} \mathrm{C}$ for $10 \mathrm{~min}$, followed by 40 cycles at $95^{\circ} \mathrm{C}$ for $15 \mathrm{sec}, 60^{\circ} \mathrm{C}$ for $30 \mathrm{sec}, 72^{\circ} \mathrm{C}$ for $30 \mathrm{sec}$ and a final incubation at $72^{\circ} \mathrm{C}$ for $10 \mathrm{~min}$. Expression levels were normalized against levels of GAPDH (for mRNAs) or U6 small nuclear RNA (for miRNAs). The relative expression levels and fold changes of each gene were calculated via the
Table II. TaqMan probe IDs used to investigate gene expression and miRNA expression levels in the present study.

\begin{tabular}{ll}
\hline Gene & TaqMan assay ID \\
\hline Wnt1 & Mm01300555_g1 \\
Wnt3 & Mm00437336_m1 \\
Wnt4 & Mm01194003_m1 \\
Wnt7a & Mm00437356_m1 \\
Wnt10a & Mm00437325_m1 \\
Wnt10b & Mm00442104_m1 \\
Tcf1 & Mm00493445_m1 \\
Lef1 & Mm00550265_m1 \\
SP1 & Mm00489039_m1
\end{tabular}

Tcf1, T-cell factor 1; Lef1, lymphoid enhancer binding factor 1; SP1, Sp1 transcription factor.

$2^{-\Delta \Delta C q}$ method (32) using Microsoft Excel 2007 (Microsoft Corporation, Redmond, WA, USA).

Alkaline phosphatase (ALP) staining. ALP substrate solution was prepared using Sigma fast red tablet sets (cat. no. F4523; Sigma-Aldrich; Merck KGaA) according to the manufacturer's instructions. BMSCs were fixed at room temperature for 10 min using 10\% neutral buffered formalin (Sigma-Aldrich; Merck KGaA) cultured in the ALP substrate solution in a $37^{\circ} \mathrm{C}$ incubator for $30 \mathrm{~min}$ and subsequently monitored using a light microscope (magnification, x100) for the development of a red reaction product, which indicated ALP activity.

miRNA prediction. TargetScan 7.0 (http://www.targetscan.org) and miRWalk 3.0 (http://mirwalk.umm.uni-heidelberg.de/) were used to identify the potential miRNAs targeting Wnt10a mRNA. In total, five putative Wnt10a-targeting miRNAs were selected based on the previously reported function of these miRNAs in bone development (33-37).

Statistical analysis. Data are presented as the mean \pm standard error of the mean. Student's t-test was calculated using Prism 7 (GraphPad Software, Inc., La Jolla, CA, USA) were used to analyze the statistical differences among different groups. $\mathrm{P}<0.05$ was considered to indicate a statistically significant difference. All experiments were repeated three times independently.

\section{Results}

Profiling of Dicer and Wnt genes during osteogenic differentiation in BMSCs. Firstly, mouse BMSCs were isolated and differentiated into multiple lineages, as previously reported (17). Adipogenic and osteoblastic markers were detected via qPCR in order to further characterize BMSCs (Fig. 1A and B). In order to establish an osteogenic differentiation model, isolated BMSCs were incubated for five days, which represented the proliferative expansion period, until they reached $100 \%$ confluency. The results revealed that there were increased levels of osteogenic markers, which suggested 

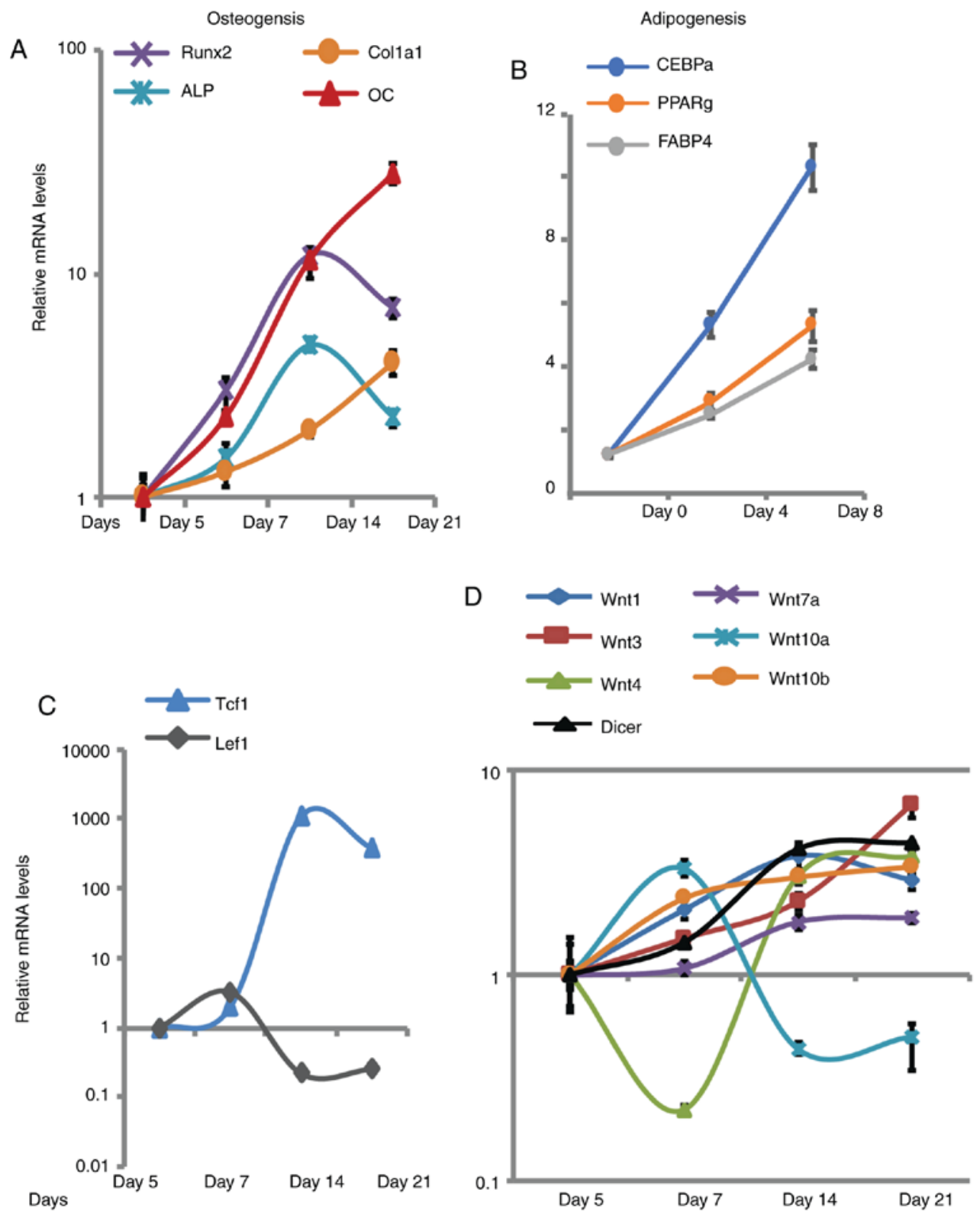

Figure 1. Wnt genes exhibited dynamic changes in expression levels during the osteogenic differentiation of BMSCs. (A) Activation of osteogenic markers were detected during the osteogenic differentiation of BMSCs. (B) Activation of adipogenic markers was detected during the adipogenic differentiation of BMSCs. (C) mRNA expression levels of Tcf-1 and Lef1 transcription factors downstream of Wnt signalling were detected in differentiating BMSCs. (D) Levels of Wnt genes and Dicer were evaluated during differentiation in BMSCs. Gene expression levels were investigated by reverse transcription-quantitative polymerase chain reaction. The values reported were compared with the determined values at day 5 of osteogenic differentiation or day 0 of adipogenic differentiation in BMSCs (set as 1). Values are presented as the mean \pm standard error of the mean $(\mathrm{n}=3)$. BMSCs, bone marrow stromal cells; ALP, akaline phosphatase; Tcf1, T-cell factor 1; Lef1, lymphoid enhancer binding factor 1; Col1a1, collagen 1a1; OC, osteocalcin; Runx2, runt-related transcription factor 2; CEBP $\alpha$, CCAAT enhancer binding protein- $\alpha$; PPAR $\gamma$, peroxisome proliferator activated receptor- $\gamma$; FABP4, fatty acid binding protein 4.

that osteogenic differentiation had been successfully induced (Fig. 1A). Important transcription factors (Tcf-1 and Lef1) represent downstream targets of the Wnt signaling pathway (15-18). The results of the present study demonstrated Tcf- 1 expression was markedly upregulated during osteogenic differentiation, whereas levels of Lef1 increased slightly between days 5-7, and then markedly decreased between days 7-21 (Fig. 1C). Similar to the majority of Wnt genes, Dicer expression was upregulated by 4 fold during the 21 days of differentiation, which suggested that increased levels of Dicer is important for osteogenic differentiation in BMSCs (Fig. 1D). To investigate the association between Wnt signaling and osteogenic differentiation, the expression levels of numerous Wnt genes were determined by RT-qPCR. Four of the six Wnt genes analysed (Wnt1, Wnt3, Wnt7a and Wnt10b) were revealed to be consistently upregulated throughout the differentiation process (Fig. 1D). Furthermore, Wnt4 was demonstrated to be downregulated between days 5-7, and upregulated between days 7-21 (Fig. 1D). However, Wnt10a levels were revealed to be increased during the proliferative period between days 5-7, and markedly decreased during the differentiation period between days 7-21. Dynamic changes of numerous Wnt genes suggested that members of the Wnt protein family exhibit different roles during the early and late differentiation stages. 
Deletion of Dicer block osteogenic differentiation, and the regulation of Wnt genes is stage-specific. To investigate the role of Dicer during osteogenic differentiation, stable transduction of BMSCs with shDicer was performed. The results revealed that $\sim 80 \%$ of Dicer was knocked down by shDicer (Fig. 2A). The results of histological analyses further demonstrated that inhibition of Dicer blocked osteogenic differentiation in BMSCs as assessed by negative ALP staining, in yellow (Fig. 2B). In BMSCs transfected with scramble shRNA [not specific (NS)], the expression levels of osteogenic differentiation markers including Runx 2 and ALP, were significantly upregulated by 4 -fold on day 14 of differentiation compared with day 7 (Fig. 2C). Therefore, the results suggested that knockdown of Dicer almost completely blocked the activation of these markers during osteogenic differentiation.

The effects of shDicer on the gene expression levels of Wnt genes in differentiating BMSCs were also investigated. At the day 5 time interval during the proliferative expansion period, knockdown of Dicer suppressed the expression levels of Wnt1 and Wnt7a by 50\%; whereas the expression levels of Wnt4, Wn10a and Tcf-1 increased by 8.1-, 2.54- and 63.5-fold, respectively (Fig. 3). Furthermore, at day 7 during differentiation, the expression levels of Wnt10b decreased by $60 \%$ in shDicer-BMSCs compared with the NS group (Fig. 3B). The expression levels of other Wnt components tested by qPCR was not altered. Transfection with shDicer increased the expression of Wnt10a by 2.5-fold in BMSCs at the 5 and 7 day time intervals compared with the NS group (Fig. 3A and B). The inhibition of Wnt1 and Wnt7a by shDicer was also observed during early differentiation on day 7; however, transfection with shDicer significantly increased the expression levels of Wnt4 and Tcf-1 by 4- and 22-fold compared with the NS group, respectively (Fig. 3B and C).

Considering that the knockdown of Dicer was revealed to be associated with the regulation of Wnt genes and Tcf- 1 at the proliferation stage (day 5) and the early differentiation stage (day 7), alterations in the expression levels of these genes in shDicer-BMSCs at the 14 and 21 day time intervals during late osteogenic differentiation were investigated. The results revealed that knockdown of Dicer suppressed the expression levels of all included Wnt genes by 50-90\%, apart from Wnt10a (Fig. 4A and B). Notably, in contrast to the activation of Tcf-1 by shDicer at the 5 and 7 day time intervals, shDicer inhibited the expression levels of Tcf- 1 by $>90 \%$ in BMSCs during the late stage of osteogenic differentiation (Fig. 4A and B), which may be due to the suppressed expression of numerous Wnt genes, resulting in the inhibition of Tcf-1. Furthermore, knockdown of Dicer was revealed to upregulate Wnt10a expression throughout the osteogenic differentiation process, and this effect was most significant at the 14 day time interval $(>5.54$ fold), which suggested that Dicer may directly regulate the Wnt10a-targeting miRNAs in order to mediate Wnt10a expression levels during the osteogenic differentiation process. To investigate whether shDicer-induced upregulation of Wnt10a is specific to osteogenic differentiation, shDicer was additionally applied to mouse fibroblasts (NIH3T3 cells). The results revealed that a total of $90 \%$ of Dicer was inhibited in transfected cells compared with the NS group (Fig. 4C). Furthermore, the expression levels of all of the examined Wnt genes, as well as Tcf-1 and Lef1, were downregulated in NIH3T3 cells

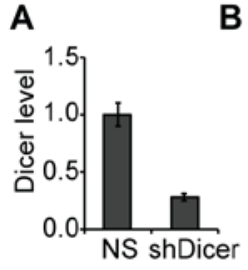

B
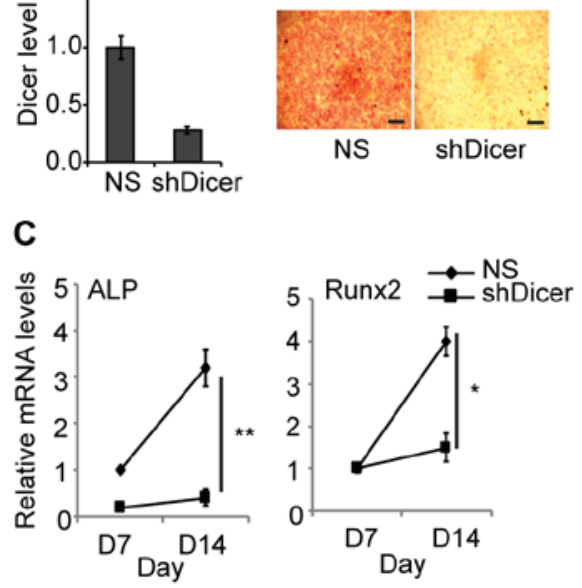

Figure 2. Knockdown of Dicer blocked osteogenic differentiation of BMSCs. (A) Dicer was downregulated by stably transducing shDicer into BMSCs. (B) ALP activity was investigated in scrambled shRNA and shDicer treated BMSCs via histochemical staining on day 14 of osteogenic differentiation. Scale bar, $100 \mu \mathrm{m}$. (C) Expression levels of Runx2 and ALP osteogenic makers were significantly inhibited in Dicer-deficient BMSCs on day 14 of differentiation compared with BMSCs that had been transfected with NS shRNA. Values are presented as the mean \pm standard error of the mean $(n=3)$. ${ }^{*} \mathrm{P}<0.05$ and ${ }^{* *} \mathrm{P}<0.01$, as indicated. BMSCs, bone marrow stromal cells; ALP, alkaline phosphatase; shRNA, short hairpin RNA; Runx2, runt related transcription factor 2; ALP, akaline phosphatase; NS, non-specific.

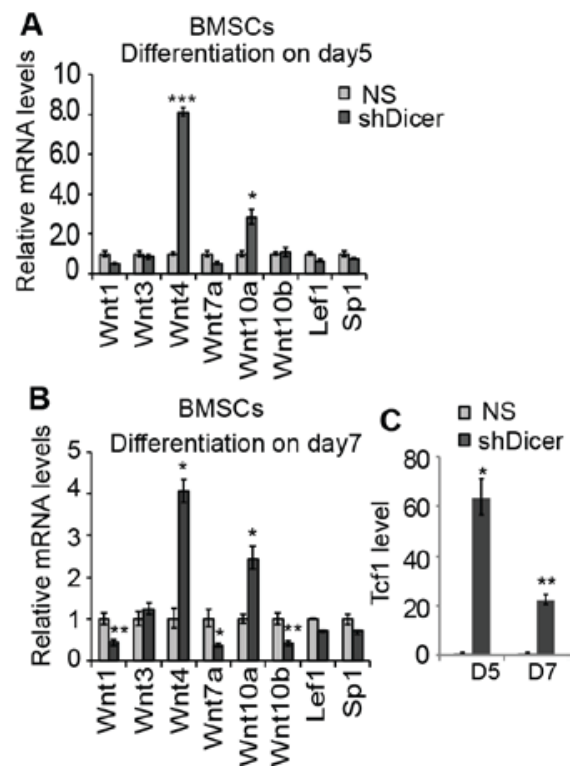

Figure 3. Differential regulation of Wnt genes via knockdown of Dicer during the early stages of osteogenic differentiation in BMSCs. (A and B) In NS shRNA- and shDicer-infected BMSCs, the expression levels of Wnt genes, Lef1 and Sp1 were detected at 5 and 7 day time intervals during differentiation. (C) Expression levels of Tcf-1 were investigated at 5 and 7 day time intervals during differentiation in shRNA-infected BMSCs. Gene expression levels were investigated by reverse transcription-quantitative polymerase chain reaction. Values are presented as the mean \pm standard error of the mean $(\mathrm{n}=3) .{ }^{*} \mathrm{P}<0.05,{ }^{* *} \mathrm{P}<0.01$ and ${ }^{* * * *} \mathrm{P}<0.001$ vs. NS group. BMSCs, bone marrow stromal cells; sh, short hairpin; NS, non-specific; Tcf-1, T-cell factor 1; Lef1, lymphoid enhancer binding factor 1; SP1, Sp1 transcription factor.

following transfection with shDicer, thus suggesting that the regulation of Wnt10a expression by Dicer is specific to the 

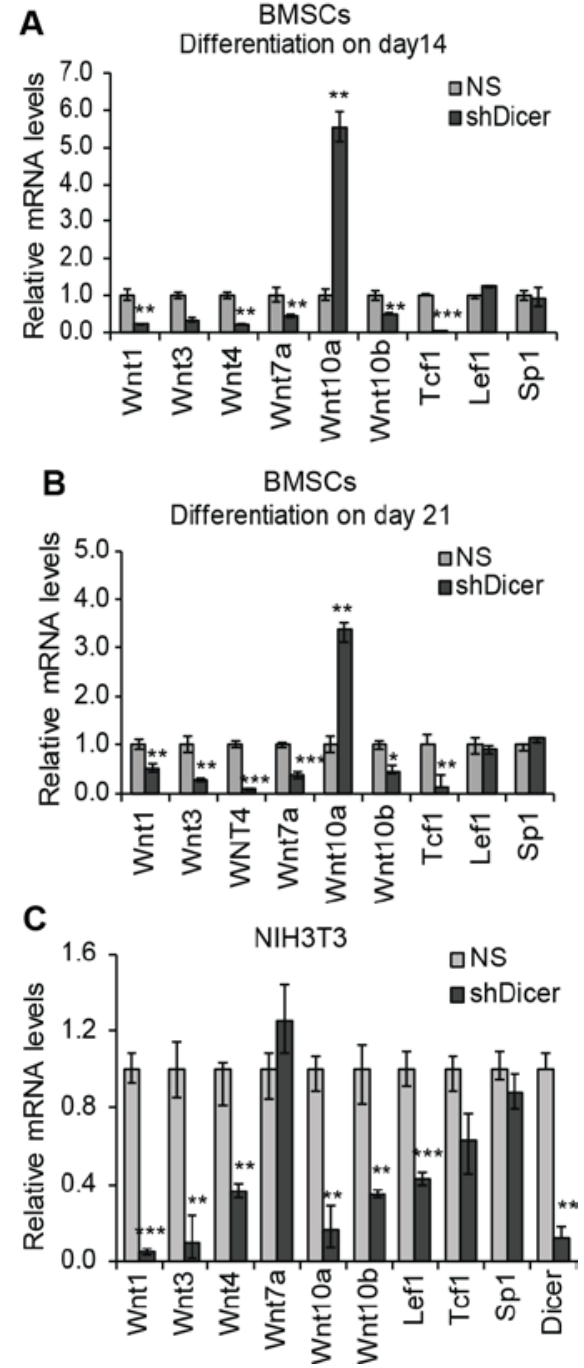

Figure 4. Regulation of Wnt genes via knockdown of Dicer during the late stage of osteogenic differentiation in BMSCs. (A and B) In BMSCs transfected with NS shRNA and shDicer, the expression levels of Wnt genes, Tcf-1, Lef1 and Sp1 were detected on days (A) 14 and (B) 21 during late osteogenic differentiation. (C) Expression levels of genes were investigated in mouse fibroblast NIH3T3 cells transfected with shDicer. Gene expression levels were investigated by reverse transcription-quantitative polymerase chain reaction. Values are presented as the mean \pm standard error of the mean $(n=3) .{ }^{*} \mathrm{P}<0.05,{ }^{* *} \mathrm{P}<0.01$ and ${ }^{* * * *} \mathrm{P}<0.001$ vs. NS group. NS, non-specific; BMSCs, bone marrow stromal cells; Tcf-1, T-cell factor 1; Lef1, lymphoid enhancer binding factor 1; sh, short hairpin; SP1, Sp1 transcription factor.

in vitro osteogenic differentiation of BMSCs and osteoblasts (Fig. 4C). Furthermore, the expression levels of SP1 were not affected by shDicer in either BMSCs or fibroblasts, thereby suggesting that the upregulation of Wnt genes, Tcf and Lef1 by Dicer is specific to osteoblasts MC3T3-1 (Figs. 3A, B and 4).

Profiling of Wht genes in osteoblast MC3T3-E1 cells. Considering that BMSCs have multi-lineage differentiation potential (17), MC3T3-E1 osteoblast cells were additionally investigated, as they have the limited potential to differentiate selectively to mature osteoblast cells. Differentiation was induced in MC3T3-E1 osteoblast cells, as assessed by a marked upregulation of Runx1, ALP and Osteocalcin expression levels (Fig. 5A). The expression levels of Wnt1, Tcf-1 and Lef1 were markedly upregulated; whereas the expression levels of Wnt4 and Wnt10a were downregulated by 50\% during the 24 days of differentiation (Fig. 5B and C). Similar to BMSC osteogenic differentiation, the expression level of Dicer was increased by 4.5-fold during the differentiation period in MC3TC-E1 cells (Fig. 5B).

Expression levels of putative Wnt10a-targeting miRNAs in BMSCs during osteogenic differentiation. Dicer is an important enzyme for the production of mature miRNAs. Therefore, deletion of Dicer may induce miRNA deficiency during osteoblast differentiation, which may decrease the suppression of Wnt10a expression by targeting miRNAs. Five putative Wnt10a-targeting miRNAs were investigated during osteogenic differentiation in BMSCs. These miRNAs were selected based on the analysis using Targetscan and miRWalk prediction software $(38,39)$. In total, four of five miRNAs (miR-28, miR-149, miR-212 and let-7b) were revealed to be markedly upregulated during osteogenic differentiation (Fig. 6A). In addition, how the deletion of Dicer affected the expression levels of the aforementioned miRNAs was investigated. The results revealed that knockdown of Dicer significantly suppressed the expression levels of the investigated miRNAs in BMSCs (Fig. 6B).

\section{Discussion}

An increasing body of evidence has demonstrated that miRNAs have important roles in bone development and homeostasis (19). Supporting these findings, it has also been previously reported that deletion of the key enzyme Dicer in mice led to marked levels of abnormal bone development (22). However, the molecular mechanisms underlying such effects require further investigation. In the present study, the dynamic changes associated with numerous Wnt genes during osteogenic differentiation in mouse BMSCs were investigated, as well as the regulation of these Wnt genes and important transcriptional factors (Tcf-1 and Lef1) by Dicer.

Extensive evidence has demonstrated that organ development, tissue homeostasis and the development of numerous diseases are associated with a number of signalling pathways, such as inflammation, Wnt/ $\beta$-catenin and metabolic dysregulation (40-42). All of the Wnt genes included in the present study have been reported to promote osteogenic differentiation (43-47). To the best of the authors' knowledge, the present study is the first to demonstrate that various Wnt genes exhibit stage-specific patterns of expression during osteogenic differentiation The activation of Wnt1 and Tcf-1 were observed in differentiating BMSCs and MC3T3-E1 osteoblasts, thus suggesting that both Wnt1 and Tcf-1 have important roles in osteoblast differentiation. The expression levels of Wnt3, Wnt7a and Wnt10b were only upregulated in differentiating BMSCs, which indicated that the upregulation of these genes may be important regarding osteogenic differentiation. Wnt 4 , a prototypical ligand for the non-canonical Wnt pathway, has been previously demonstrated to promote osteoblast differentiation of mesenchymal stem cells (48). Furthermore, in an in vivo transgenic model, Wnt 4 has been revealed to promote bone formation and block bone resorption (49). The results of the present study revealed that the expression levels of Wnt4 

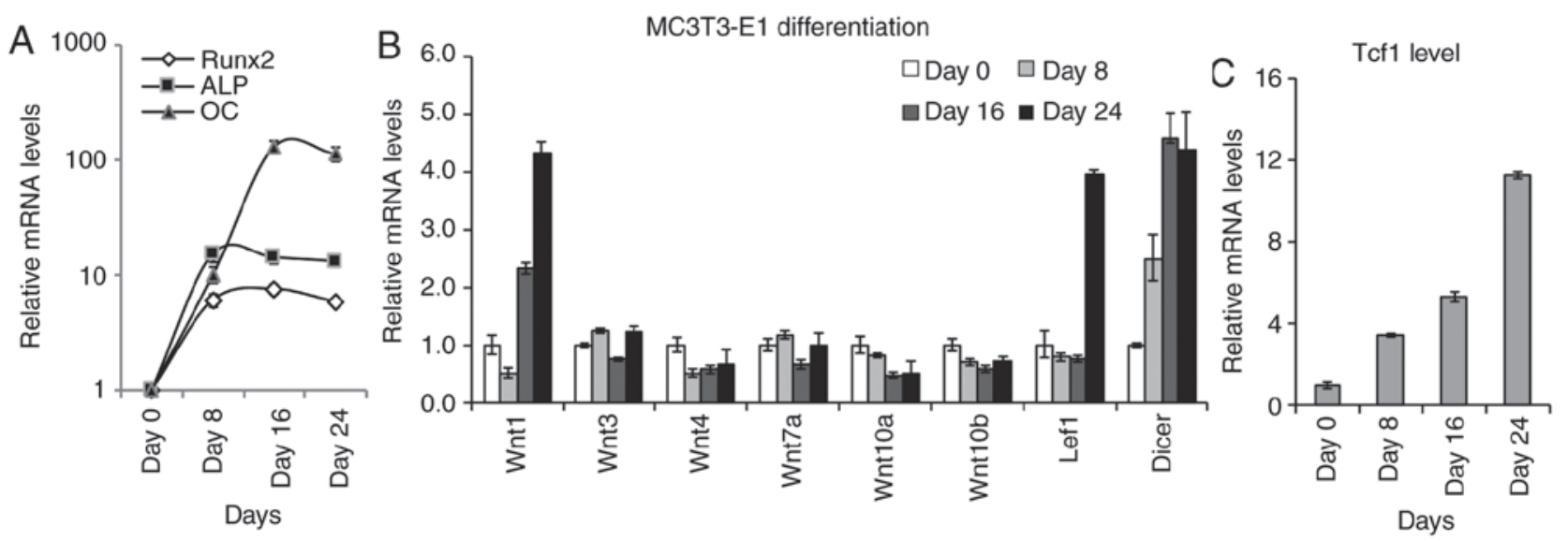

Figure 5. Expression levels of Wnt genes, Tcf1 and Lef1 during osteoblast differentiation. (A) Osteogenic markers (Runx2, ALP and OC) were upregulated during differentiation of MC3T3-E1 cells. (B) Wnt genes, Lef1 and Dicer expression levels were investigated at the 24 day time intervals during osteoblast differentiation. (C) Tcf-1 levels was detected during osteoblast differentiation. Values are presented as the mean \pm standard error of the mean ( $\mathrm{n}=3$ ). Tcf-1, T-cell factor 1; Lef1, lymphoid enhancer binding factor 1; Runx2, runt related transcription factor 2; ALP, akaline phosphatase; OC, osteocalcin; NS, non-specific.
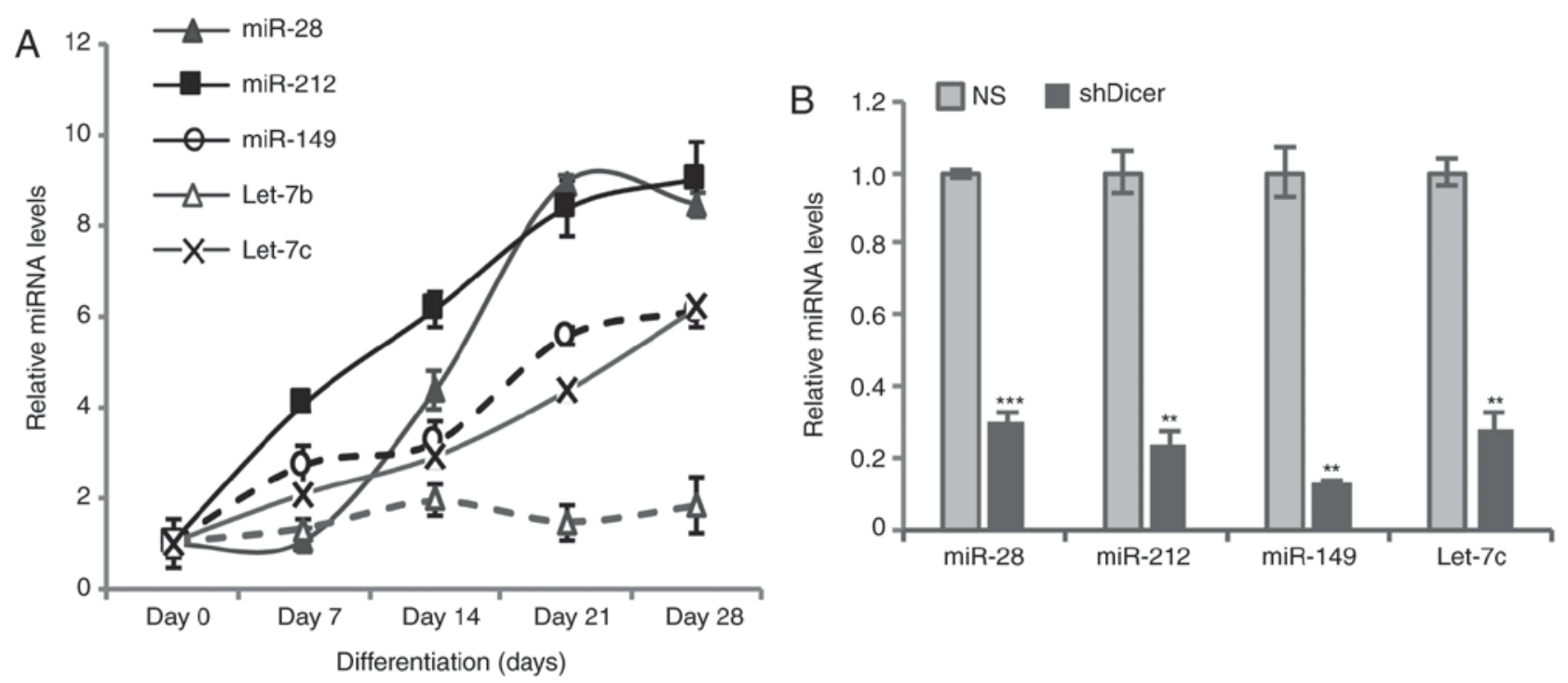

Figure 6. Dicer regulates Wnt10a-targeting miRNA expression. (A) Five putative Wnt10a-targeting miRNAs were investigated during BMSC osteogenic differentiation. The values presented are relative to the values determined on day 0 (set as 1). (B) Four upregulated Wnt10a-targeting miRNAs were detected in BMSCs transfected with shRNA. Values are presented as the mean \pm standard error of the mean $(n=3) .{ }^{* *} \mathrm{P}<0.01$ and ${ }^{* * *} \mathrm{P}<0.001$ vs. NS group. miRNA, microRNA; sh, short hairpin; BMSCs, bone marrow stromal cells; NS, non-specific; miR, microRNA.

were decreased during the proliferative expansion period and then significantly increased during the differentiation stage. Notably, Wnt 4 expression was decreased during MC3T-E1 differentiation in the present study. These results indicated that the upregulation of Wnt4 may be involved in osteoblast proliferation; however, increased levels of Wnt4 may not be essential for osteoblast differentiation. In differentiating BMSCs, Wnt10a exhibited opposite expression patterns to Wnt4. The result suggested that Wnt10a may be associated with the proliferative expansion of BMSCs, but not with differentiation. This hypothesis was further supported by the fact that Wnt10a expression levels were downregulated during the differentiation of osteoblasts in the present study.

In osteogenic and osteoblast differentiation models, Dicer was upregulated, which suggested that it has an important role during this biological process. Consistent with previous studies, marked blockage of osteogenic differentiation in shDicer-BMSCs was demonstrated in the present study (22). The differential regulation of the included Wnt genes via knockdown of Dicer is coordinated well with their dynamic expression pattern during differentiation. For example, during the early stage of differentiation (days 5-7), Wnt4 and Tcf-1 levels were relatively low in undifferentiated BMSCs. Knockdown of Dicer significantly increased Wnt4 and Tcf-1 levels on days 5 and 7, which may be involved in cell proliferation. During the late stage of differentiation, deficiency of Dicer resulted in the downregulation of Wnt4 and Tcf- 1 expression, delaying or blocking osteogenic differentiation. The present results suggested that Wnt4 and Tcf- 1 may serve stage-specific roles.

Upregulation of Wnt10a via knockdown of Dicer may represent the only protein included in the present study that 
directly regulates miRNA deficiency. Activation of Wnt10a could switch adipogenesis to osteogenesis (50); however, its function is not clear during osteogenic differentiation. In the present study, numerous putative Wnt10a-targeting miRNAs, which were upregulated during osteoblast differentiation and inhibited via knockdown of Dicer, were determined. In the future, the mechanisms associated with the miRNAs targeting Wnt10a during osteogenesis require further investigation. Previous studies identified that, besides directly targeting Wnt genes, a number of Dicer-dependent miRNAs may target inhibitors of Wnt genes, including Dickkopf-related protein 1 and secreted frizzled-related protein 1 (51). Deletion of Dicer leads to a decreased maturation of miRNAs that may target various Wnt inhibitors, resulting in the downregulation of Wnt genes.

In conclusion, the dynamic expression pattern of numerous Wnt genes during osteogenic differentiation was investigated in the present study, as well as the direct and indirect regulation of these genes by Dicer. Considering the important roles of Wnt signaling in bone development, the results suggested that the inhibition of Wnt signalling may have a major effect on defective bone formation in Dicer-knockout or -knockdown models. miRNAs generated by Dicer may represent important regulators of Wnt signaling during osteogenic differentiation.

\section{Acknowledgements}

Not applicable.

\section{Funding}

No funding was received.

\section{Availability of data and materials}

All data generated or analysed during this study are included in this published article.

\section{Authors' contributions}

HYW conceived the present study, provided resources, was involved in the investigations, analysis and visualization of data, supervised the study and wrote the manuscript. RB, TS and FX performed the experiments and analysed the data. All authors have read and approved the final manuscript.

\section{Ethics approval and consent to participate}

The present study was granted ethical approval by the Fourth Hospital of Hebei Medical University (Shijiazhuang, China).

\section{Patient consent for publication}

Not applicable.

\section{Competing interests}

The authors declare that they have no competing interests.

\section{References}

1. Lian JB, Stein GS, Javed A, van Wijnen AJ, Stein JL, Montecino M, Hassan MQ, Gaur T, Lengner CJ and Young DW: Networks and hubs for the transcriptional control of osteoblastogenesis. Rev Endocr Metab Disord 7: 1-16, 2006.

2. Soltanoff CS, Yang S, Chen W and Li YP: Signaling networks that control the lineage commitment and differentiation of bone cells. Crit Rev Eukaryot Gene Expr 19: 1-46, 2009.

3. Maupin KA, Droscha CJ and Williams BO: A comprehensive overview of skeletal phenotypes associated with alterations in wnt/ $\beta$-catenin signaling in humans and mice. Bone Res 1: 27-71, 2013.

4. Fahiminiya S, Majewski J, Mort J, Moffatt P, Glorieux FH and Rauch F: Mutations in WNT1 are a cause of osteogenesis imperfecta. J Med Genet 50: 345-348, 2013.

5. Laine CM, Joeng KS, Campeau PM, Kiviranta R, Tarkkonen K, Grover M, Lu JT, Pekkinen M, Wessman M, Heino TJ, et al: WNT1 mutations in early-onset osteoporosis and osteogenesis imperfecta. N Engl J Med 368: 1809-1816, 2013.

6. Zheng HF, Tobias JH, Duncan E, Evans DM, Eriksson J, Paternoster L, Yerges-Armstrong LM, Lehtimäki T, Bergström U, Kähönen M, et al: WNT16 influences bone mineral density, cortical bone thickness, bone strength, and osteoporotic fracture risk. PLoS Genet 8: e1002745, 2012.

7. Kato M, Patel MS, Levasseur R, Lobov I, Chang BH, Glass DA II, Hartmann C, Li L, Hwang TH, Brayton CF, et al: Cbfa1-independent decrease in osteoblast proliferation, osteopenia, and persistent embryonic eye vascularization in mice deficient in Lrp5, a Wnt coreceptor. J Cell Biol 157: 303-314, 2002.

8. Riddle RC, Diegel CR, Leslie JM, Van Koevering KK, Faugere MC, Clemens TL and Williams BO: Lrp5 and Lrp6 exert overlapping functions in osteoblasts during postnatal bone acquisition. PLoS One 8: e63323, 2013.

9. Cui Y, Niziolek PJ, MacDonald BT, Zylstra CR, Alenina N, Robinson DR, Zhong Z, Matthes S, Jacobsen CM, Conlon RA, et al: Lrp5 functions in bone to regulate bone mass. Nat Med 17: 684-691, 2011.

10. Lu W, Yamamoto V, Ortega B and Baltimore D: Mammalian Ryk is a Wnt coreceptor required for stimulation of neurite outgrowth. Cell 119: 97-108, 2004.

11. He X, Semenov M, Tamai K and Zeng X: LDL receptor-related proteins 5 and 6 in Wnt/beta-catenin signaling: Arrows point the way. Development 131: 1663-1677, 2004.

12. Chen DQ, Cao G, Chen H, Liu D, Su W, Yu XY, Vaziri ND, Liu XH, Bai X, Zhang L and Zhao YY: Gene and protein expressions and metabolomics exhibit activated redox signaling and wnt/ $\beta$-catenin pathway are associated with metabolite dysfunction in patients with chronic kidney disease. Redox Biol 12: 505-521, 2017.

13. Chen L, Chen DQ, Wang M, Liu D, Chen H, Dou F, Vaziri ND and Zhao YY: Role of RAS/Wnt/ $\beta$-catenin axis activation in the pathogenesis of podocyte injury and tubulo-interstitial nephropathy. Chem Biol Interact 273: 56-72, 2017.

14. Komiya $\mathrm{Y}$ and Habas R: Wnt signal transduction pathways. Organogenesis 4: 68-75, 2008.

15. Hovanes K, Li TW, Munguia JE, Truong T, Milovanovic T, Lawrence Marsh J, Holcombe RF and Waterman ML: Beta-catenin-sensitive isoforms of lymphoid enhancer factor-1 are selectively expressed in colon cancer. Nat Genet 28: 53-57, 2001.

16. Behrens J, von Kries JP, Kuhl M, Bruhn L, Wedlich D, Grosschedl R and Birchmeier W: Functional interaction of beta-catenin with the transcription factor Lef-1. Nature 382: 638-642, 1996.

17. Wang M, Chen DQ, Chen L, Cao G, Zhao H, Liu D, Vaziri ND, Guo Y and Zhao YY: Novel inhibitors of the cellular RAS components, poricoic acids, target Smad3 phosphorylation and Wnt $/ \beta$-catenin pathway against renal fibrosis. Br J Pharmacol 175: 2689-2708, 2018.

18. Chen H, Yang T, Wang MC, Chen DQ, Yang Y and Zhao YY: Novel RAS inhibitor 25-O-methylalisol $\mathrm{F}$ attenuates epithelial-to-mesenchymal transition and tubulo-interstitial fibrosis by selectively inhibiting TGF- $\beta$-mediated Smad3 phosphorylation. Phytomedicine 42: 207-218, 2018.

19. Husain A and Jeffries MA: Epigenetics and bone remodeling. Curr Osteoporos Rep 15: 450-458, 2017.

20. Hassan MQ, Tye CE, Stein GS and Lian JB: Non-coding RNAs: Epigenetic regulators of bone development and homeostasis. Bone 81: 746-756, 2015.

21. Ha M and Kim VN: Regulation of microRNA biogenesis. Nat Rev Mol Cell Biol 15: 509-524, 2014. 
22. Gaur T, Hussain S, Mudhasani R, Parulkar I, Colby JL, Frederick D, Kream BE, van Wijnen AJ, Stein JL, Stein GS, et al: Dicer inactivation in osteoprogenitor cells compromises fetal survival and bone formation, while excision in differentiated osteoblasts increases bone mass in the adult mouse. Dev Biol 340: 10-21, 2010.

23. Oommen S, Otsuka-Tanaka $\mathrm{Y}$, Imam $\mathrm{N}$, Kawasaki $\mathrm{M}$, Kawasaki K, Jalani-Ghazani F, Anderegg A, Awatramani R, Hindges R, Sharpe PT and Ohazama A: Distinct roles of microRNAs in epithelium and mesenchyme during tooth development. Dev Dyn 241: 1465-1472, 2012.

24. Li J, Zhang Y, Zhao Q, Wang J and He X: MicroRNA-10a influences osteoblast differentiation and angiogenesis by regulating $\beta$-catenin expression. Cell Physiol Biochem 37: 2194-2208, 2015.

25. Tang X, Lin J, Wang G and Lu J: MicroRNA-433-3p promotes osteoblast differentiation through targeting DKK1 expression. PLoS One 12: e0179860, 2017.

26. Long H, Sun B, Cheng L, Zhao S, Zhu Y, Zhao R and Zhu J: miR-139-5p represses BMSC osteogenesis via targeting wnt//-catenin signaling pathway. DNA Cell Biol 36: 715-724, 2017.

27. Kureel J, John AA, Prakash R and Singh D: MiR 376c inhibits osteoblastogenesis by targeting Wnt3 and ARF-GEF-1facilitated augmentation of beta-catenin transactivation. J Cell Biochem 119: 3293-3303, 2018

28. Cao F, Zhan J, Chen X, Zhang K, Lai R and Feng Z: miR-214 promotes periodontal ligament stem cell osteoblastic differentiation by modulating Wnt $/ \beta$-catenin signaling. Mol Med Rep 16: 9301-9308, 2017.

29. Bae JS, Gutierrez S, Narla R, Pratap J, Devados R, van Wijnen AJ, Stein JL, Stein GS, Lian JB and Javed A: Reconstitution of Runx2/Cbfa1-null cells identifies a requirement for BMP2 signaling through a Runx2 functional domain during osteoblast differentiation. J Cell Biochem 100: 434-449, 2007.

30. Xu S, De Becker A, Van Camp B, Vanderkerken K and Van Riet I: An improved harvest and in vitro expansion protocol for murine bone marrow-derived mesenchymal stem cells. J Biomed Biotechnol 2010: 105940, 2010.

31. Wang M, Chen DQ, Wang MC, Chen H, Chen L, Liu D, Zhao H and Zhao YY: Poricoic acid ZA, a novel RAS inhibitor, attenuates tubulo-interstitial fibrosis and podocyte injury by inhibiting TGF- $\beta$ /Smad signaling pathway. Phytomedicine 36: 243-253, 2017.

32. Livak KJ and Schmittgen TD: Analysis of relative gene expression data using real-time quantitative PCR and the 2(-Delta Delta C(T)) method. Methods 25: 402-408, 2001.

33. Dernowsek JA, Pereira MC, Fornari TA, Macedo C, Assis AF, Donate PB, Bombonato-Prado KF, Passos-Bueno MR and Passos GA: Posttranscriptional interaction between miR-450a-5p and miR-28-5p and STAT1 mRNA triggers osteoblastic differentiation of human mesenchymal stem cells. J Cell Biochem 118 4045-4062, 2017.

34. Dole NS and Delany AM: MicroRNA variants as genetic determinants of bone mass. Bone 84: 57-68, 2016.

35. de la Rica L, García-Gómez A, Comet NR, Rodríguez-Ubreva J, Ciudad L, Vento-Tormo R, Company C, Álvarez-Errico D, García M, Gómez-Vaquero C and Ballestar E: NF-кB-direct activation of microRNAs with repressive effects on monocyte-specific genes is critical for osteoclast differentiation. Genome Biol 16: 2, 2015.
36. Yuan $\mathrm{H}$, Zhao $\mathrm{H}$, Wang J, Zhang $\mathrm{H}$, Hong L, Li $\mathrm{H}$, Che $\mathrm{H}$ and Zhang Z: MicroRNA let-7c-5p promotes osteogenic differentiation of dental pulp stem cells by inhibiting lipopolysaccharide-induced inflammation via HMGA2/PI3K/Akt signal blockade. Clin Exp Pharmacol Physiol 21: 1440-1681, 2018.

37. Wei J, Li H, Wang S, Li T, Fan J,Liang X, Li J, Han Q, Zhu L, Fan L and Zhao RC: let-7 enhances osteogenesis and bone formation while repressing adipogenesis of human stromal/mesenchymal stem cells by regulating HMGA2. Stem Cells Dev 23: 1452-1463, 2014.

38. Dweep H, Sticht C, Pandey P and Gretz N: miRWalk-database: Prediction of possible miRNA binding sites by 'walking' the genes of three genomes. J Biomed Inform 44: 839-847, 2011.

39. Agarwal V, Bell GW, Nam JW and Bartel DP: Predicting effective microRNA target sites in mammalian mRNAs. ELife 4: e05005, 2015.

40. Nusse $\mathrm{R}$ and Clevers $\mathrm{H}$ : Wnt/ $\beta$-catenin signaling, disease, and emerging therapeutic modalities. Cell 169: 985-999, 2017.

41. Zhao YY, Cheng XL, Vaziri ND, Liu S and Lin RC: UPLC-based metabonomic applications for discovering biomarkers of diseases in clinical chemistry. Clin Biochem 47: 16-26, 2014.

42. Chen L, Su W, Chen H, Chen DQ, Wang M, Guo Y and Zhao YY: Proteomics for biomarker identification and clinical application in kidney disease. Adv Clin Chem 85: 91-113, 2018.

43. Cawthorn WP, Bree AJ, Yao Y, Du B, Hemati N, Martinez-Santibañez G and MacDougald OA: Wnt6, Wnt10a and Wnt10b inhibit adipogenesis and stimulate osteoblastogenesis through a $\beta$-catenin-dependent mechanism. Bone 50: 477-489, 2012.

44. Shao JS, Cheng SL, Pingsterhaus JM, Charlton-Kachigian N, Loewy AP and Towler DA: Msx2 promotes cardiovascular calcification by activating paracrine Wnt signals. J Clin Invest 115: 1210-1220, 2005

45. Adamska M, MacDonald BT, Sarmast ZH, Oliver ER and Meisler MH: En1 and Wnt7a interact with Dkk1 during limb development in the mouse. Dev Biol 272: 134-144, 2004.

46. Minear S, Leucht P, Jiang J, Liu B, Zeng A, Fuerer C, Nusse R and Helms JA: Wnt proteins promote bone regeneration. Sci Transl Med 2: 29ra30, 2010

47. Rauch F: The brains of the bones: How osteocytes use WNT1 to control bone formation. J Clin Invest 127: 2539-2540, 2017.

48. Chang J, Sonoyama W, Wang Z, Jin Q, Zhang C, Krebsbach PH, Giannobile W, Shi S and Wang CY: Noncanonical Wnt-4 signaling enhances bone regeneration of mesenchymal stem cells in craniofacial defects through activation of p38 MAPK. J Biol Chem 282: 30938-30948, 2007.

49. Yu B, Chang J, Liu Y, Li J, Kevork K, Al-Hezaimi K, Graves DT, Park NH and Wang CY: Wnt4 signaling prevents skeletal aging and inflammation by inhibiting nuclear factor- $\kappa \mathrm{B}$. Nat Med 20: 1009-1017, 2014

50. Chen YS, Wu R, Yang X, Kou S, MacDougald OA, Yu L, Shi H and Xue B: Inhibiting DNA methylation switches adipogenesis to osteoblastogenesis by activating Wnt10a. Sci Rep 6: 25283, 2016.

51. Kawano Y and Kypta R: Secreted antagonists of the Wnt signalling pathway. J Cell Sci 116: 2627-2634, 2003. 